Centro di Studi di Genetica umana dell'Università di Milano

Direttore: Prof. L. Gianferrari

\title{
Angelo Serra
}

\section{Considerazioni intorno alla metodologia della ricerca sull'associazione fenotipica tra gruppi sanguigni e malattie nelle popolazioni umane}

Numerose e piuttosto severe critiche sono state rivolte, particolarmente da qualche antropologo (Manuila I958 a, I958 b), alle ricerche sull'associazione tra gruppi sanguigni e malattie nelle popolazioni umane ed alle conclusioni e ipotesi da quelle derivate. Il punto fondamentale della critica verte sulla metodologia: si sottolinea l'insufficienza dei campioni studiati, l'inadeguatezza delle serie di controllo, l'invariabilità dei risultati anche nei piccoli campioni, la limitata validità delle conclusioni ricavate dall'esame delle differenze nella distribuzione dei gruppi sanguigni e si avverte che differenze dell'ordine del $5 \%$ o anche ı $\%$ tra le frequenze di dati gruppi sanguigni in due popolazioni non possono essere rivelatrici di una diversa distribuzione dei gruppi stessi, anche se sono statisticamente significative.

Tutte queste difficoltà erano già presenti ad Aird, ai suoi collaboratori (1953, I954), ed a parecchi altri - anche se non a tutti gli altri -- che si occuparono di questo problema, nè furono trascurate nell'analisi ed interpretazione dei dati. Sembra quindi per lo meno inappropriata e per qualche aspetto insufficientemente ponderata $\mathrm{o}$, talvolta, inconsistente l'impostazione stessa della critica, in realtà poco costruttiva, del Manuila. Ad ogni modo, esigendo l'importanza genetica e medica (McConnell, 1957) della ricerca la maggiore accuratezza possibile, saranno qui brevemente esaminati alcuni elementi metodologici che debbono essere tenuti in considerazione nei due tempi, campionamento e analisi statistica, in cui si svolge l'indagine. 


\section{Il campionamento}

La risposta alla questione se vi sia o no una certa dipendenza tra l'essere affetti da una determinata malattia e l'appartenere ad un dato gruppo sanguigno è dedotta dall'esito della verifica dell'ipotesi statistica iniziale che non esista alcuna associazione tra gruppi sanguigni e malattie: se questa ipotesi si deve respingere resta confermata l'ipotesi alternativa, cioè l'associazione.

Detta verifica importa il confronto di due campioni: un campione di soggetti affetti da una data malattia o «campione sperimentale », ed un campione di controllo. Precisamente nella loro scelta va tenuto presente un fondamentale elemento metodologico, dal quale dipende in gran parte il risultato definitivo di tutta l'analisi statistica; i campioni devono essere rappresentativi, sufficienti, omogenei.

Rappresentativi. I campioni debbono rispecchiare le caratteristiche della popolazione da cui sono estratti, potendo differirne solo per cause accidentali. Il campionamento dovrebbe perciò essere casuale.

E senza dubbio difficile provare che siano tali le serie di pazienti ospedalizzate, che costituiscono ordinariamente il campione sperimentale; d'altra parte è anche difficile provare che non lo siano, specialmente quando sono grandi. In pratica, ai fini della ricerca di cui si parla, queste serie possono essere ritenute valide se sono rispettate le seguenti condizioni:

I. assenza di selezione al ricovero riguardo ai caratteri clinici della forma morbosa, alla condizione sociale, alla regione d'origine e all'età.

2. assenza di selezione nella determinazione di gruppo: si avrebbe selezione invece se la tipizzazione fosse eseguita, ad esempio a scopo trasfusionale, solo per le forme di particolare gravità o da sottoporre a trattamento chirurgico.

3. assenza di selezione al rilevamento, comprendendo tutti i casi entro i limiti stabiliti per la ricerca.

Più facile è raccogliere dei campioni di controllo rappresentativi della popolazione generale da cui provengono i malati. Serie di successivi donatori volontari - non professionali - presso gli ospedali o i Centri trasfusionali costituiscono il materiale più adatto allo scopo. Lo studio di Buckwalter (1958) in merito ne dà seria garanzia.

Sufficienti. I campioni dovrebbero essere di grandezza tale da ridurre tanto i limiti (al $95 \%$ di probabilità) della variazione casuale delle frequenze dei gruppi sanguigni o del rischio di malattia, da permettere la dimostrazione di differenze statisticamente significative tra le frequenze di dati gruppi nelle due popolazioni o tra le incidenze della malattia in soggetti di diverso gruppo sanguigno anche quando dette differenze sono piccole. Secondo Fraser Roberts (1956) il numero dei soggetti che si richiederebbe perchè un campione mostri, con il $95 \%$ di probalità, una differenza significativa al livello dell' $1 \%$, salirebbe da 600 a 1250,2400 e 6500 quando l'incidenza relativa di una malattia in un dato gruppo fosse rispettivamente $\mathrm{I}, 4$; I,3; I,2; I, I.

D'altra parte va pure notato che quanto più un campione è piccolo tanto meno 
Tabella I - Effetto della dimensione del campione

\begin{tabular}{|c|c|c|c|c|c|c|c|}
\hline \multirow{3}{*}{ Campione } & \multicolumn{4}{|c|}{ Numero dei soggetti } & \multirow{3}{*}{$\begin{array}{c}\text { I. R. } \\
\text { nel gruppo } \\
\text { A }\end{array}$} & \multirow{3}{*}{$\chi^{2}$} & \multirow{3}{*}{$P$} \\
\hline & \multicolumn{2}{|c|}{$\begin{array}{l}\text { con carcinoma } \\
\text { del collo } \\
\text { dell'utero }\end{array}$} & \multicolumn{2}{|c|}{ controllo } & & & \\
\hline & A & $\mathrm{O}$ & A & $\mathrm{O}$ & & & \\
\hline 1. $194^{8-1} 95^{6}$ & 60 & 40 & $9^{8}$ I & I006 & 1,43 & 3,06 & $0,10, \mathrm{P}_{>0,05}$ \\
\hline 2. $1957-195^{8}$ & 24 & 35 & $9^{8 I}$ & I 006 & 0,70 & 1,72 & $0,20>P>0,10$ \\
\hline
\end{tabular}

I. Beolchini e coll. (1957); 2. Beolchini (1958).

facilmente riesce rappresentativo. Una prova sperimentale è presentata nella tabella I. Mentre dai 103 casi di cancro dell'utero (portio), ospedalizzati e tipizzati nel periodo I948-1956 nell'Istituto Provinciale di Maternità di Milano, risulterebbe che la frequenza del cancro è sensibilmente maggiore nel gruppo A che nel gruppo O, dai 59 casi del periodo I957 - agosto $195^{8}$ apparirebbe semplicemente il contrario: è infatti notevolmente minore nel gruppo $A$ che nel gruppo $O$. Sorge logicamente il dubbio della rappresentatività dei due campioni. Ed infatti l'eterogeneità delle due serie di pazienti rispetto alla distribuzione dei gruppi A e O è tale $\left(\chi_{[1]}^{2}=3,90 ; 0,02<\mathrm{P}<0,05\right)$ che, pur provenendo presumibilmente da un'identica popolazione di cancerose, non si possono considerare come campioni casuali di essa.

Omogenei. I) I campioni dourebbero essere internamente omogenei.

Per il campione sperimentale, questa proprietà si riduce essenzialmente alla omogeneità clinica: cioè unica dovrebbe essere l'entità nosologica della forma che si studia. Si osservino le tabelle $2 \mathrm{a}$ e $2 \mathrm{~b}$.

E chiaro che l'incidenza relativa (I. R.) del cancro gastrico in un dato gruppo sanguigno non è tendenzialmente uniforme se si considerano a parte le sue principali localizzazioni: i carcinomi antropilorici e del cardias prevalgono nettamente nel gruppo A; i carcinomi del corpo nel gruppo O. Naturalmente una simile eterogeneità fa risentire $\mathrm{i}$ suoi effetti quando si analizza tutto il complesso dei carcinomi: secondo la frequenza dei carcinomi dell'una o dell'altra sede inclusi nel campione, si potrà riuscire o meno a dimostrare una significativa incidenza in un dato gruppo. Si nota infatti - come si attenderebbe - che a mano a mano che diminuisce la percentuale dei carcinomi del corpo nelle tre serie $(47,4 \%$ in quella del Billington, $38,5 \%$ in quella del Cappellini e $34,5 \%$ in quella del Jennings) si passa dalla non significatività alla significatività della incidenza relativa nel gruppo A per il totale dei casi.

Il fatto della variabilità dell'associazione gruppo sanguigno-malattia secondo la localizzazione di questa, variabilità che come si è dimostrato per il cancro gastrico

1 Test di significatività secondo il metodo di Woolf. 


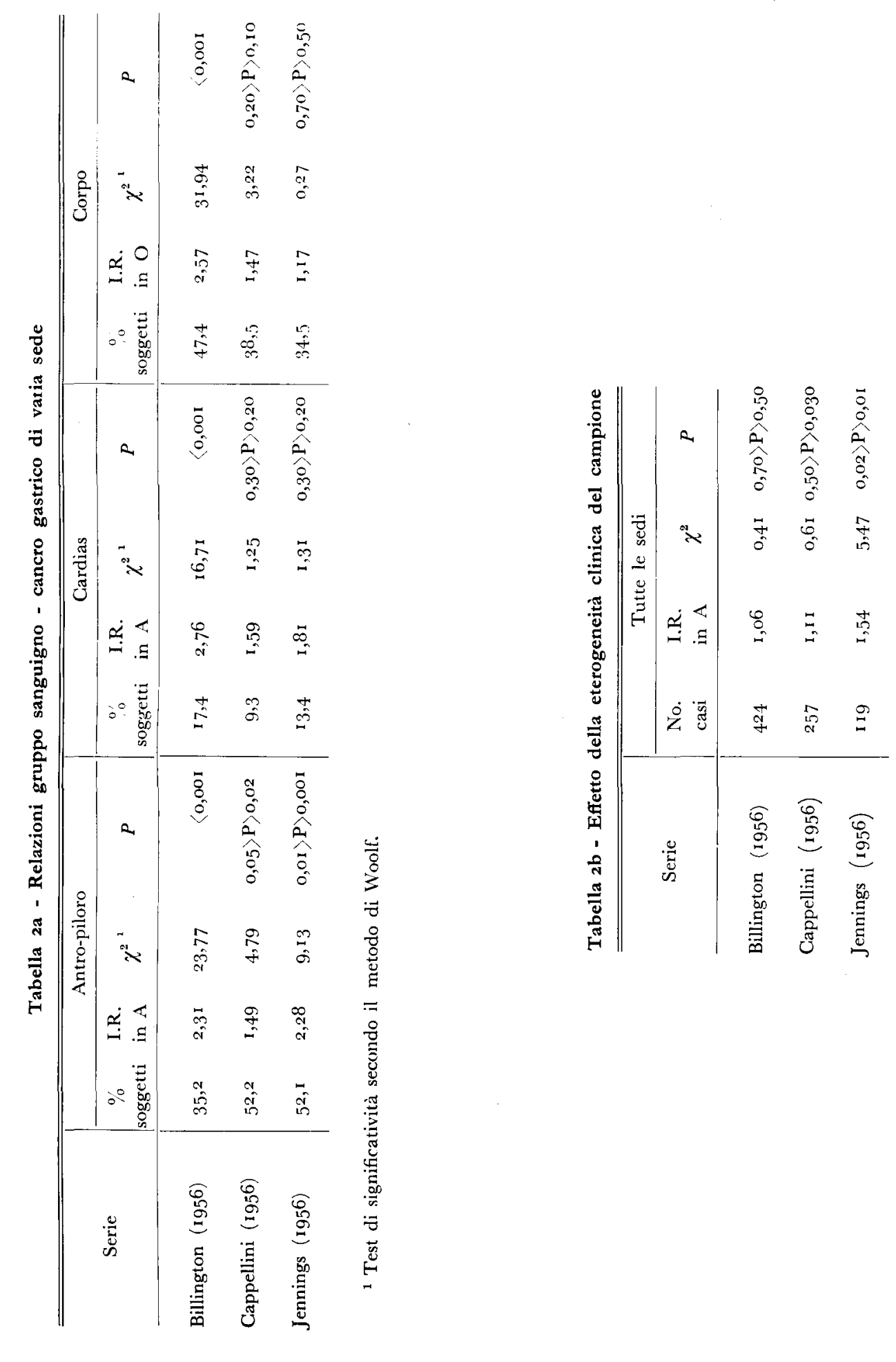


si può spingere oltre a quanto era già stato verificato per l'ulcera peptica (Clarke e coll. 1955; Bentall 1956), impone quindi un'attenzione particolare nello stabilire l'entità nosologica di una forma morbosa per questo specifico studio.

Lo stesso fatto conduce inoltre a pensare che a questo tipo di eterogeneità interna siano da attribuire - almeno in parte - i risultati negativi di Wallace (I954), Speiser (I956) e Benda (I957) circa la significativa prevalenza dei carcinomi gastrici nel gruppo $\mathrm{A}$, benchè anche in queste tre serie il gruppo A tenda a predominare; e così pure le contrastanti conclusioni di Beolchini e coll. (1957), di Liotta e coll. (1957) e di Cavallini (1958) sul cancro dell'utero.

Per il campione di controllo l'omogeneità interna esige l'esclusione di soggetti che tendono ad introdurre differenze sistematiche nella distribuzione dei gruppi sanguigni relativamente a quella della popolazione generale. Buckwalter ( 1958 ) prova ad esempio che l'inclusione di donatori professionali nella serie dei volontari - essendo in gran parte di gruppo $\mathrm{O}$ - porta ad una deviazione molto significativa dalla distribuzione effettiva. In base a questa esigenza si comprende pure come sarebbe preferibile non utilizzare quale controllo serie di pazienti ricoverati negli stessi ospedali dove sono raccolti $\mathrm{i}$ casi di malattia in esame. I dati in corso di elaborazione da parte del Comitato per lo studio della distribuzione geografica dei gruppi sanguigni in Italia (Barigozzi e coll. 1958), costituiranno - ritengo - il miglior materiale di controllo per le singole regioni e provincie italiane; mentre per parecchie località, soprattutto grandi città, questo è fornito già dai dati di Morganti (I957).

2) I campioni sperimentale e controllo dovrebbero essere omogenei tra loro.

Qui è soprattutto necessaria la omogeneità etnica: poichè soltanto se questa esiste è logico porre quale ipotesi iniziale l'ipotesi «zero ».

Sarebbe perciò conveniente conoscere l'origine tanto dei malati che dei controlli in modo da poter eliminare ogni sensibile eterogeneità di questo tipo.

E pure desiderabile l'omogeneità per età, sesso e condizioni sociali, benchè non si sia ancora pienamente dimostrato che la distribuzione dei gruppi sanguigni vari con l'una o l'altra di queste variabili ed esistano anzi forti indizi in contrario - almeno per l'età e il sesso - quali i risultati negativi ottenuti da Buckwalter (I958) su un materiale di 49.979 donatori.

\section{L'analisi statistica \\ A - Metodi classicr}

Raccolti i campioni, per raggiungere una decisione sulla significatività o meno dell'associazione in esame, bisogna ricorrere all'analisi statistica che, come si è già accennato, consiste nel verificare l'ipotesi «zero».

Sia dal punto di vista medico che genetico interessa stabilire prima di tutto l'esistenza di una associazione tra $\mathrm{i}$ fenotipi dei vari sistemi di antigeni ed una data malattia. Ciò può essere fatto portando l'analisi su campioni singoli o su campioni combinati. 
L'analisi su campioni singoli. Sono stati proposti e impiegati particolarmente due metodi.

I. Il metodo di Aird e coll. (1953, I954): Consiste essenzialmente nel determinare la probabilità di trovare per caso, se fosse vera l'ipotesi zero, una differenza tra le frequenze di un dato gruppo (o di un insieme di gruppi o dei vari gruppi) uguale o maggiore di quella osservata tra $i$ due campioni; da cui si deduce la significatività della differenza e quindi dell'associazione. Allo scopo si può utilizzare la distribuzione « $t »$ o la distribuzione $\chi^{2}$.

E sufficiente averlo accennato perchè, se è molto valido per singoli campioni abbastanza grandi, è svantaggioso quando lo si applichi a campioni combinati, cioè costituiti da serie di dati raccolti in aree diverse. Questo metodo infatti, che si basa sul criterio della differenza di frequenze, è sensibile alla diversa distribuzione dei gruppi sanguigni nelle diverse aree. Una esemplificazione è data nella tabella 3 .

Tabella 3 - Effetto della distribuzione dei gruppi sanguigni sui risultati dell'analisi della differenza di frequenze

\begin{tabular}{|c|c|c|c|c|c|c|c|c|c|}
\hline & $H$ & $\kappa$ & $h$ & $k$ & $h|n-H| \mathcal{N}$ & $v(d)$ & $s_{d}$ & $t$ & $P$ \\
\hline I. & $\begin{array}{l}5.000 \\
(0,50)\end{array}$ & $\begin{array}{l}5.000 \\
(0,50)\end{array}$ & $\begin{array}{c}\text { I00 } \\
(0,66)\end{array}$ & $\begin{array}{c}5^{\circ} \\
(0,33)\end{array}$ & 0,1667 & $0,0015^{0}$ & $0,03_{3} 3$ & 4,353 & $\langle 0,001$ \\
\hline 2. & $\begin{array}{l}7.000 \\
(0,70)\end{array}$ & $\begin{array}{l}3.000 \\
(0,30)\end{array}$ & $\begin{array}{c}140 \\
(0,82)\end{array}$ & $\begin{array}{c}3^{0} \\
(0,18)\end{array}$ & 0,1235 & 0,00087 & 0,0295 & 4,186 & $\langle 0, O O I$ \\
\hline 3. & $\begin{array}{l}9.000 \\
(0,90)\end{array}$ & $\begin{array}{r}1.000 \\
(0,10)\end{array}$ & $\begin{array}{c}\text { I80 } \\
(0,95)\end{array}$ & $\begin{array}{c}10 \\
(0,05)\end{array}$ & 0,0474 & 0,00026 & 0,0160 & 2,963 & $\mathrm{O}, \mathrm{OI}\langle\mathrm{P}\langle\mathrm{O}, \mathrm{OOI}$ \\
\hline
\end{tabular}

Si abbia una serie di 3 campioni, ciascuno costituito da un sottocampione sperimentale e uno controllo. Nel campione controllo $H$ è il numero dei soggetti di gruppo A, $K$ di gruppo $\mathrm{O}$; nel campione sperimentale $h$ sono i soggetti di gruppo A e $k$ di gruppo $\mathrm{O}$. $H+K=\mathcal{N} ; h+k=n$. Si faccia l'ipotesi che nelle tre aree da cui provengono questi campioni il rischio di ammalarsi per i soggetti dei due gruppi A e $\mathrm{O}$ resti costante: e precisamente sia $2 \%$ per $\mathrm{i}$ soggetti di gruppo A e $\mathrm{I} \%$ per $\mathrm{i}$ soggetti di gruppo O. Allora nella popolazione I, dove la frequenza del gruppo A e $\mathrm{O}$ sono uguali, su ad esempio 5000 soggetti sani di gruppo A e 5000 di gruppo $\mathrm{O}$ se ne attenderanno rispettivamente 100 e 50 malati; nella popolazione 2 , dove le frequenze dei due gruppi sono l'una 0,70 e l'altra 0,30 , su 7000 soggetti sani di gruppo A e 3000 di gruppo $\mathrm{O}$ se ne attenderanno rispettivamente i 40 e 30 malati; nella popolazione 3 , dove le frequenze dei due gruppi sono l'una o,9o e l'altra o, ı, su gooo soggetti sani di gruppo $\mathrm{A}$ e Iooo di gruppo $\mathrm{O}$ se ne attenderanno rispettivamente r8o e ro malati. Facendo ora la differenza tra le frequenze dei soggetti di gruppo A nei corrispondenti campioni sperimentale e controllo si vede che le differenze sono rispettivamente $17 \%$, I $2 \%$, e $5 \%$, pur essendo uguale ovunque l'incidenza della 
malattia tra i soggetti di detto gruppo. E ciò si risente nel test di significatività, essendo il livello della significatività statistica della differenza di frequenze una funzione soprattutto della grandezza della differenza stessa.

Naturalmente resta in tal modo introdotto un elemento estraneo di eterogeneità nella serie dei campioni, che aumenta la varianza complessiva del campione combinato ed è difficilmente separabile nell'analisi da altri fattori intrinseci di eterogeneità. Come si rileva dall'esempio l'entità dell'eterogeneità varia e, quando i campioni provengono da aree di una stessa nazione dove è abbastanza omogenea la distribuzione dei gruppi, il metodo sarebbe utilizzabile. Siccome però le ulteriori ricerche potranno richiedere la combinazione di dati su popolazioni con frequenze geniche di gruppo assai diverse, sembra conveniente uniformare il metodo anche per l'analisi dei campioni singoli.

2) Il metodo di Woolf-Haldane (I955-1956): Consiste essenzialmente nel determinare la probabilità di trovare per caso, se fosse vera l'ipotesi "zero ", un'incidenza relativa di malati in un dato gruppo o insieme di gruppi uguale o maggiore di quella osservata; da cui si deduce la significatività dell'incidenza e quindi dell'associazione.

Tabella 4. - Analisi dell'associazione tra gruppi sanguigni e malattie secondo il metodo di Woolf e Haldane

\begin{tabular}{|c|c|c|}
\hline \multicolumn{3}{|c|}{ CAMPIONI SINGOLI } \\
\hline Woolf & & Haldane \\
\hline \multirow{2}{*}{$x=h K / k H$} & \multirow{2}{*}{$x=$} & $(2 h+\mathbf{I})(2 \boldsymbol{K}+\mathbf{I})$ \\
\hline & & $(2 k+1)(2 H+\mathbf{I})$ \\
\hline$y=\ln x$ & $y=$ & $\ln x$ \\
\hline \multirow{2}{*}{$v(y)=\mathbf{I} / h+\mathbf{I} / k+\mathbf{I} / H+\mathbf{I} / K$} & \multirow{2}{*}{$V(y)=$} & $(H+h)(K+k)$ \\
\hline & & $\mathcal{N} n(\mathcal{N}+n)$ \\
\hline$\chi^{2}=y^{2} / v(y)$ & $c=$ & $y / 1 \quad V(y)$ \\
\hline
\end{tabular}

\section{CAMPIONI COMBINATI}

$$
\begin{aligned}
w & =\mathbf{r} / v(y) \\
y_{w} & =\sum w y / \sum_{w} \\
\chi_{[1]}^{2} & =\left(\sum w y\right)^{2} / \Sigma_{w}=y_{w}^{2} \sum_{w} \\
\chi_{[c-1]}^{2} & =\sum w y^{2}-\left(\sum w y\right)^{2} / \sum w
\end{aligned}
$$

2 Nel derivare questa espressione della varianza sembra che l'A. sia incorso in una svista. Nell'applicazione che se ne farà (tabella 5), per evitare errori di valutazione, sarà usata la formula corretta che dovrebbe essere : $\frac{(\mathcal{N}+n)^{2}(\mathcal{N}+n-I)}{\mathcal{N} n(H+h)}$. Ringrazio il Prof. J. A. Fraser Roberts che ha voluto gentilmente attirare la mia attenzione su questo punto. 
Se ad esempio la frequenza di una particolare malattia nel gruppo A è del $30 \%$ maggiore che nel gruppo $\mathrm{O}$, è cioè $\mathrm{I}, 30$ rispetto alla frequenza nel gruppo $\mathrm{O}$ fatta uguale ad I, si esegue il test della sua significativa deviazione da I — valore che invece si attenderebbe se fossero ugualmente colpiti i soggetti dei due gruppi - per mezzo della distribuzione $\chi^{2}$ o per mezzo della distribuzione normale $c$.

Nella tabella 4 è indicato il procedimento di calcolo. Secondo Woolf, se $h$ e $k$ sono rispettivamente $\mathrm{i}$ soggetti di gruppo $\mathrm{A}$ e $\mathrm{O}$ nel campione sperimentale ed $H$ e $K$ i soggetti di gruppo corrispondente nel campione controllo, $x=h K / k H$ è una stima efficiente del rischio relativo di malattia nel gruppo A rispetto a quello $O$. Per facilità di calcolo, operando sul logaritmo naturale di tal rapporto $y$, se ne stima la varianza $v(y)$ con cui si confronta $y^{2}$ ottenendo il $\chi^{2}$.

Questo metodo ha due vantaggi a) di permettere un'analisi di campioni combinati in cui la varianza complessiva non è aumentata dall'eterogeneità spuria, introdotta col metodo precedente come un effetto della diversa distribuzione dei gruppi sanguigni nelle diverse popolazioni; e b) di presentare in $x$ un valore di diretto significato biologico, indicando esso il rischio relativo di malattia per soggetti di un dato gruppo.

Tuttavia tale procedimento è valido per grandi campioni. Ordinariamente invece i campioni singoli non sono molto grandi. Le stime perciò sono generalmente viziate.

Tabella 5 - Confronto della sensibilità dei metodi di Woolf e Haldane

\begin{tabular}{|c|c|c|c|c|c|c|}
\hline \multirow{2}{*}{ Serie } & \multicolumn{3}{|c|}{ Metodo di Woolf } & \multicolumn{3}{|c|}{ Metodo di Haldane } \\
\hline & $\begin{array}{c}\text { I.R. } \\
\text { nel gruppo }\end{array}$ & $c$ & $P$ & $\begin{array}{c}\text { I.R. } \\
\text { nel gruppo }\end{array}$ & c & $P$ \\
\hline $\begin{array}{l}\text { 1. Ulcera gastrica } \\
\text { (300 casi) }\end{array}$ & $\begin{array}{c}\mathrm{O} \\
\mathrm{I}, \mathrm{1} 8\end{array}$ & $\mathrm{I}, 400$ & $0,20>P>0,10$ & $\begin{array}{c}\mathrm{O} \\
\mathrm{I}, \mathrm{I} 8\end{array}$ & $\mathrm{I}, 405$ & $0,20>P>0,1_{0}$ \\
\hline $\begin{array}{l}\text { 2. Ulcera duodenale } \\
(1642 \text { casi })\end{array}$ & $\begin{array}{l}\text { I, } 17 \\
\text { A }\end{array}$ & $2,74^{\circ}$ & $\mathrm{O}, \mathrm{OI}>\mathrm{P}>\mathrm{O}, \mathrm{OOI}$ & $\begin{array}{l}\text { I, I } 7 \\
\text { A }\end{array}$ & $2,75^{2}$ & $0,0 \mathbf{I}>\mathrm{P}>0,00 \mathbf{I}$ \\
\hline $\begin{array}{l}\text { 3. Cancro gastrico } \\
\text { (66 casi) }\end{array}$ & $\mathbf{1}, 95$ & 2,553 & $0,02>\mathrm{P}>0,0 \mathrm{I}$ & $\mathrm{I}, 93$ & 2,663 & $0,0 \mathrm{I}>\mathrm{P}>\mathrm{O}, \mathrm{OO} \mathrm{I}$ \\
\hline $\begin{array}{l}\text { 4. Cancro gastrico } \\
(87 \text { casi })\end{array}$ & $\mathrm{I}, 79$ & $2,65^{1}$ & $0, \mathrm{OI}>\mathrm{P}>0, \mathrm{OOI}$ & $\mathrm{I}, 79$ & $2,65 \mathrm{I}$ & $0, \mathrm{OI}>\mathrm{P}>0, \mathrm{OOI}$ \\
\hline $\begin{array}{l}\text { 5. Cancro gastrico } \\
(184 \text { casi })\end{array}$ & 1,39 & 2,200 & $0,05>P>0,02$ & I,39 & 2,201 & $0,05>\mathrm{P}>0,02$ \\
\hline $\begin{array}{l}\text { 6. Cancro gastrico } \\
\text { (300 casi) }\end{array}$ & $\mathrm{I}, 44$ & $2,55^{\circ}$ & $0,02>P>0,01$ & $\mathrm{I}, 44$ & 2,554 & $0,02>\mathrm{P}>0,0 \mathrm{I}$ \\
\hline $\begin{array}{l}\text { 7. Cancro gastrico } \\
\text { (799 casi) }\end{array}$ & 1,15 & I,966 & $\stackrel{\infty}{0,05}$ & $\mathrm{I}, \mathrm{I} 5$ & 1,960 & $\cong 0,05$ \\
\hline
\end{tabular}


Haldane (1956), partendo dallo studio di Woolf (1955), ha dedotto teoricamente le correzioni da introdurre in generale nella stima e nel test di significatività del logaritmo di un rapporto di frequenze. Applicate dallo stesso Haldane al nostro problema, esse sono indicate nella tabella 4 , dove $\mathcal{N}=H+K$ e $n=h+k$.

Nella tabella 5 un confronto dei risultati del metodo di Woolf, applicato senza e con le correzioni suggerite da Haldane ad alcune casistiche, mette in evidenza la diversa sensibilità dei due procedimenti. Si nota soprattutto la maggior sensibilità del procedimento di Haldane nel caso di piccoli campioni dell'ordine di $n=50 \div 100$. Nei casi invece in cui si ha un numero maggiore di osservazioni, le stime ottenute col metodo di Woolf sono da ritenere sufficientemente precise.

L'analisi su campioni combinati. Il grande numero di casi è una necessità per questo tipo di ricerche. Un mezzo per raggiungerlo si ha nella combinazione di campioni singoli.

L'analisi deve allora tener conto della diversità del numero dei soggetti nei diversi campioni. Bisogna quindi attribuire (tabella 4) a ciascun $y$ un peso $w$, assunto come l'inverso della varianza; la media ponderata degli $y$ pesati $y_{\mathrm{w}}$ darà una stima del logaritmo naturale del rischio relativo medio, ed il $\chi^{2}$ per un grado di libertà valuterà la significatività della deviazione dell'incidenza relativa media da $\mathrm{I}$.

Tabella 6 - Analisi della associazione tra gruppi sanguigni e carcinoma gastrico in diverse aree italiane (metodo di Woolf)

\begin{tabular}{|c|c|c|c|c|c|c|c|}
\hline \multirow[b]{2}{*}{ Area } & \multicolumn{4}{|c|}{ Numero dei soggetti } & \multirow{2}{*}{$\begin{array}{c}\text { I. R. } \\
\text { nel gruppo } \\
\text { A }\end{array}$} & \multirow[b]{2}{*}{$\chi^{2}$} & \multirow[b]{2}{*}{$P$} \\
\hline & $\begin{array}{c}\text { con ca. } \\
\text { A }\end{array}$ & $\begin{array}{c}\text { astrico } \\
0\end{array}$ & $\mathrm{~A}^{\mathrm{CO}}$ & ollo & & & \\
\hline I. Forlì & I 36 & $9^{2}$ & 545 & 636 & 1,72 & I 3,65 & $\langle 0,001$ \\
\hline 2. Genova & 202 & I 87 & 604 & 688 & 1,23 & $3,2 \mathrm{I}$ & $0,10>\mathrm{P}>0,05$ \\
\hline 3. Genova-Riv. & 47 & $3^{I}$ & 604 & 688 & $1,7 \mathrm{I}$ & 5,16 & $0,02>\mathrm{P}>0,01$ \\
\hline 4. Milano & 327 & 262 & $98 \mathrm{I}$ & I.006 & 1,22 & 4,43 & $0,05>P>0,02$ \\
\hline 5. Milano & 44 & 22 & 37.806 & $3^{6.837}$ & $\mathrm{I}, 93$ & $6,3 \mathrm{I}$ & $0,02>P>0,01$ \\
\hline 6. Napoli & I 42 & I I 7 & 374 & 444 & $\mathrm{I}, 44$ & 6,47 & $0,02>\mathrm{P}>0,0 \mathrm{I}$ \\
\hline 7. Palermo & 97 & 63 & 327 & $44^{8}$ & 2,10 & I 7,55 & $\langle 0,001$ \\
\hline 8. Perugia & I94 & I 88 & 3.127 & 3.163 & $\mathrm{I}, 04$ & 0,16 & $0,70>P>0,5^{\circ}$ \\
\hline
\end{tabular}

\begin{tabular}{lccc} 
& \multicolumn{3}{c}{ Analisi del $\chi^{2}$} \\
Totale & g. 1. & $\chi^{2}$ & $P$ \\
Deviazione & 8 & 56,80 & \\
Eterogeneità & $\mathbf{1}$ & $37, \mathrm{r} 6$ & $\langle$ o, oo I \\
& 7 & $\mathbf{1 9}, 64$ & o, or $\rangle$ P $\rangle$ o, oo I
\end{tabular}

I.: Maltoni e coll. (I956); 2.: Cappellini e coll. (r956); 3.: Spandonari e coll. (r957); 4.: Beolchini e coll. (1958a); 5.: Mangani (1957); 6.: Pollara e coll. (1957); 7.: Di Carlo e coll. (1957); 8.: Benda e coll. (1957). 
Nella tabella 6 è riportato un esempio di analisi di campioni combinati: vi sono riunite le ricerche, a noi note, compiute in Italia sulla relazione tra carcinoma gastrico e gruppi sanguigni. L'analisi è stata eseguita con il metodo di Woolf corretto secondo Haldane. Se ne deduce che la deviazione da I del rischio relativo medio è estremamente significativa $\left(\chi_{[1]}^{2}=63, \mathrm{I} 7 ; P<0,00 \mathrm{I}\right)$. Sembrerebbe quindi provato anche dai dati italiani la prevalenza del cancro gastrico nei soggetti di gruppo A.

Tuttavia per essere completa l'analisi dei campioni combinati richiede la determinazione del $\chi^{2}$ per l'eterogeneità, $\chi^{2}{ }_{[\mathrm{c-r}]}$, che permette di valutare se esiste tra $i$ diversi campioni una differenza significativa nella stessa entità del rischio relativo. Lo si ottiene sottraendo dal $\chi^{2}$ totale, dato dalla somma dei singoli $\chi^{2}$, il componente che riguarda la deviazione. Nel nostro caso esso indica che l'eterogeneità è molto significativa: fatto che non è stato dimostrato nella serie inglese relativa ai carcinomi gastrici (F. Roberts, I 956) bensì nella serie delle ulcere duodenali (F. Roberts, 1956). Non sembra quindi logico nè statisticamente esatto stimare il rischio relativo medio ed i suoi limiti fiduciali.

Sebbene sia difficile, in base ai dati pubblicati, dare una spiegazione della elevata eterogeneità dimostrata, si può ritenere però che buona parte di essa sia dovuta alla insufficienza dei campioni sperimentali di alcune aree accompagnata dalla loro non rappresentatività e alla loro eterogeneità clinica nel senso precedentemente esposto. Prima di formulare altre ipotesi sarebbe necessario rimuovere le precedenti cause di eterogeneità. D'altro lato, anche se a questo stadio dell'analisi dal punto di vista statistico si potrebbero sollevare dei dubbi sopra la significatività della deviazione da I per il complesso dei dati, non pare logico negarla essendo essa evidente nei tre campioni abbastanza grandi di Milano, Napoli e Palermo, mentre l'eccezione di Perugia non è strana quando pure si accettasse un'incidenza relativa media di 1,33 .

\section{B - Metodo sequenziale}

Oltre ai metodi classici di analisi statistica, un altro è utilizzabile con notevole facilità e con molto vantaggio $\mathrm{da}$ chi può svolgere la ricerca direttamente nelle cliniche. È il cosi detto «test sequenziale », la cui teoria fu elaborata da Wald (I947) ed il cui merito principale sta nella riduzione sensibile e talvolta anche notevole, rispetto agli ordinari test, del numero delle osservazioni richieste per raggiungere una decisione attendibile. Ciò è collegato con una sua caratteristica fondamentale che consiste nel fatto che l'analisi sequenziale prescinde da una determinata grandezza del campione per il raggiungimento della decisione. Mentre infatti nei metodi classici di prova la decisione di accettare o meno un'ipotesi statistica è subordinata alla costanza del numero $n$ delle osservazioni, essendo « un procedimento di verifica semplicemente una regola che specifica per ogni possibile campione di grandezza $n$ se l'ipotesi debba essere respinta o accettata » (Wald, p. I4), nell'analisi sequenziale invece la decisione dipende, ad ogni nuova osservazione, dai risultati delle osservazioni fatte sino a quel momento. Il richiamo di alcuni elementi della 
teoria generale nel formularne l'applicazione allo studio dei rapporti tra gruppi sanguigni e malattie, già proposta al II Symposium Internazionale di Genetica Medica (Cresseri e coll., I957), gioverà a valutare meglio il significato e la portata del test.

La popolazione dei soggetti colpiti da una particolare malattia sia costituita da $\mathcal{N}$ individui: comprendendo essa tutti i soggetti affetti da quella malattia in una data area, la si può considerare sufficientemente grande di modo che ogni singola osservazione è praticamente indipendente dall'altra. Ciascuno degli $\mathcal{N}$ soggetti, riferendoci al sistema $\mathrm{ABO}$, può appartenere ad uno dei quattro gruppi $\mathrm{A}, \mathrm{O}, \mathrm{B}, \mathrm{AB}$; tuttavia in base ad una semplificazione ragionevole e già accettata anche nell'uso dei metodi classici, si supponga che la popolazione sia suddivisa in due classi soltanto: in particolare, soggetti A e $\mathrm{O}$ oppure $\mathrm{A}$ e non-A oppure $\mathrm{O}$ e non-O. Limitandoci per concretezza alla suddivisione in soggetti A e O, la caratteristica « gruppo sanguigno » di un dato soggetto, venuto a caso sotto osservazione all'atto della visita ambulatoriale o del ricovero, è una variabile casuale $x$ che può assumere due valori: ascriviamo il valore $\mathrm{I}$ ai soggetti di gruppo A e valore o ai soggetti di gruppo $\mathrm{O}$. $p=n / \mathcal{N}$, dove $n$ è il numero dei soggetti di gruppo $\mathrm{A}$, è la probabilità che l'esito dell'osservazione sia $x=\mathrm{I} ; \mathrm{I}-p=(\mathcal{N}-n) / \mathcal{N}$ è la probabilità complementare che sia $x=0$.

In realtà il parametro $p$ è ignoto. Qui però interessa soltanto decidere se $p$ è uguale alla probabilità $p_{0}$ che un soggetto dello stesso gruppo appartenente alla popolazione controllo ha di cadere sotto osservaziọne, oppure ne è significativamente maggiore: in altri termini se la frequenza dei soggetti di gruppo A è uguale nclle due popolazioni (ipotesi zero: $\mathrm{H}_{\mathbf{o}}$ ) oppure è significativamente maggiore nella popolazione sperimentale (ipotesi alternativa: $H_{1}$ ). Evidentemente $\mathrm{i}$ parametri possibili sono molti ed il loro spazio può essere ripartito in tre zone (fig. I): la prima in cui si trovano i valori $p$ consistenti con l'ipotesi zero, per cui questa si preferisce accettare (zona di accettazione); la seconda in cui si trovano $i$ valori $p$ non consistenti per eccesso con l'ipotesi zero, per cui questa si preferisce respingere (zona di repulsione); la terza in cui si trovano i valori $p$ per cui non è preferibile nè l'accettazione nè la repulsione dell'ipotesi zero (zona di indifferenza). Si possono così stabilire due valori $p_{0}$ e $\underline{p}_{1}$ tali che va considerato errore accettare $\mathrm{H}_{0}$ soltanto se $p \geq p_{1}$ e respingere $\mathrm{H}_{\mathrm{o}}$ soltanto se $p=p_{0}$. Il valore $p_{0}$ è fissato dal valore noto della popolazione di controllo: nel nostro caso la frequenza del gruppo $\mathrm{A}$; il valore $p_{\mathbf{1}}$ è scelto ad arbitrio ma in modo tale che rappresenti una frequenza che si giudichi notevolmente più clevata di quella della popolazione controllo. Si tratta quindi di verificare l'ipotesi $\mathrm{H}_{0}, p=p_{0}$, contro l'alternativa $\mathrm{H}_{1}, p=p_{1}$, sulla base di un campionamento casuale. Siccome l'osservazione non si estende a tutta la popolazione è possibile giungere ad una decisione falsa sul valore del parametro $p$. Si deve fissare allora il rischio che si desidera tollerare di fare decisioni sbagliate: $\alpha$ è il rischio che si desidera tollerare di respingere $\mathrm{H}_{0}$ quando è vera; $\beta$ è $\mathrm{il}$ rischio che si desidera tollerare di respingere $\mathrm{H}_{1}$ quando è vera. Non essendoci motivi per assegnare due livelli diversi si porrà $\alpha=\beta=0,05$ o o,or, che corrispondono ai comuni livelli della significatività nei test ordinari. 
Scelte in tal modo le quattro quantità $p_{0}, p_{1}, \alpha$ e $\beta$, che non sono un problema statistico, si passa al procedimento di verifica.

Il test sequenziale più adatto è il test del rapporto delle probabilità (probability ratio test), di forza $(\alpha, \beta)$, che può essere delineato in generale nel seguente modo: $f(x, p)$ indichi la distribuzione di probabilità della variabile casuale $x$ quando il parametro da cui dipende è $p$. Nell'ipotesi $\mathrm{H}_{\mathrm{o}}, p=p_{0}$, la distribuzione è data da $f\left(x, p_{0}\right)$; nell'ipotesi $\mathrm{H}_{1}$ da $f\left(x, p_{1}\right)$.

Si facciano successive osservazioni di $x$, indicate $x_{1}, x_{2}, x_{i} \ldots \ldots x_{n}$.

Per ogni valore intero positivo $m$, cioè ad ogni successivo stadio dell'esperimento, si può calcolare la probabilità di avere un dato campione $\left(x_{1}, x_{2} \ldots x_{\mathrm{m}}\right)$ nella supposizione che sia vera $\mathrm{H}_{1} \circ \mathrm{H}_{0}$. Esse sono:

se è vera $\mathrm{H}_{1}$ e

$$
P_{1 m}=f\left(x_{1}, p_{1}\right) f\left(x_{2}, p_{1}\right) \ldots f f\left(x_{m}, p_{1}\right)
$$

se è vera $\mathrm{H}_{0}$.

$$
P_{\mathrm{om}}=f\left(x_{1}, p_{0}\right) f\left(x_{2}, p_{0}\right) \ldots \ldots f\left(x_{\mathrm{m}}, p_{\mathrm{o}}\right)
$$

Ad ogni successiva osservazione si calcola il rapporto $P_{1 \mathrm{~m}} / P_{\mathrm{om}}$, e si applica la seguente regola fondamentale:

si accetta l'ipotesi $\mathrm{H}_{1}$;

$$
\begin{aligned}
& \text { se } \quad \frac{\beta}{\mathrm{I}-a}<\frac{P_{\mathrm{m}}}{P_{\mathrm{om}}}<\frac{\mathrm{I}-\beta}{\alpha} \text { l'osservazione si continua; } \\
& \text { se } \quad \frac{P_{1 \mathrm{~m}}}{P_{\mathrm{om}}} \geq \frac{\mathrm{I}-\beta}{\alpha} \quad \text { l'osservazione si sospende e }
\end{aligned}
$$

$$
\text { se } \quad \frac{P_{1 \mathrm{~m}}}{P_{\mathrm{om}}} \leq \frac{\beta}{\mathrm{I}-\alpha} \quad \text { l'osservazione si sospende e }
$$

si accetta l'ipotesi $\mathrm{H}_{\mathrm{o}}$.

Venendo al nostro problema la distribuzione di $x$ è data dalla funzione $f(x, p)$ che è definita solo per due valori di $x$, cioè $x=\mathrm{I}$ quando il soggetto è di gruppo A e $x=0$ quando il soggetto è di gruppo $\mathrm{O}$. Se $p$ è la probabilità che sia $x=\mathrm{I}$, sarà $f\left(x_{\mathrm{i}}, p\right)=p$ per $x_{\mathrm{i}}=\mathrm{I}$ e $f\left(x_{\mathrm{i}}, p\right)=\mathrm{I}-p$ per $x_{\mathrm{i}}=0$. La probabilità di ottenere un campione uguale all'osservato $\left(x_{1}, x_{2} \ldots \ldots x_{\mathrm{m}}\right)$ è data allora da

$$
P=p^{\mathrm{A} m}(\mathrm{I}-p)^{m-\mathrm{A}_{\mathrm{m}}}
$$

dove $A_{m}$ e $m-A_{m}$ indicano rispettivamente il numero dei soggetti $A$ e $O$ nelle prime $m$ unità osservate. Se è vera l'ipotesi $\mathrm{H}_{1}, p=p_{1}$, sarà

se è vera l'ipotesi $\mathrm{H}_{0}, p=p_{0}$, sarà

$$
P_{\mathrm{Im}}=p_{1}^{\mathrm{A}_{\mathrm{m}}}\left(\mathrm{I}-p_{1}\right)^{m-\mathrm{A}_{\mathrm{m}}}
$$

$$
P_{\text {om }}=p_{0}{ }^{\mathrm{A} m}\left(\mathbf{I}-p_{\mathrm{o}}\right)^{m-\mathrm{A}_{\mathrm{m}}}
$$

Per eseguire il test si dovrebbe perciò calcolare ad ogni successiva osservazione il rapporto $P_{1 \mathrm{~m}} / P_{\text {om }}$ che, passando ai logaritmi, diventa:

$$
\log \frac{P_{1 \mathrm{~m}}}{P_{\mathrm{om}}}=\mathrm{A}_{\mathrm{m}} \log \frac{p_{1}}{p_{\mathrm{o}}}+\left(m-\mathrm{A}_{\mathrm{m}}\right) \log \frac{\mathrm{I}-p_{1}}{\mathrm{I}-p_{\mathrm{o}}}
$$


Fino a quando

$$
\log \frac{\beta}{\mathrm{I}-a}<\log \frac{P_{1 \mathrm{~m}}}{P_{\mathrm{om}}}<\log \frac{\mathrm{I}-\beta}{\alpha}
$$

si continua l'osservazione; ma all'osservazione per cui diventa

$$
\log \frac{P_{1 \mathrm{~m}}}{P_{\mathrm{om}}} \geq \log \frac{\mathrm{I}-\beta}{\alpha}
$$

si sospende l'esperimento e si accetta l'ipotesi $\mathrm{H}_{1}$; se invece

$$
\log \frac{P_{1 \mathrm{~m}}}{P_{\mathrm{om}}} \leq \log \frac{\beta}{\mathrm{I}-\alpha}
$$

si sospende l'esperimento e si accetta l'ipotesi $\mathrm{H}_{\mathrm{o}}$.

Non c'è tuttavia bisogno di eseguire tutti questi calcoli che, del resto, con alcuni accorgimenti non sarebbero più laboriosi di quelli delle prove ordinarie. Il test si può eseguire graficamente: ed è per questo motivo che diventa molto facile il suo impiego.

$\mathrm{Si}$ dimostra infatti che le tre precedenti disuguaglianze sono rispettivamente equivalenti alle seguenti:

$$
\frac{\log \frac{\beta}{\mathrm{I}-\alpha}}{\log \frac{p_{1}}{p_{0}}-\log \frac{\mathrm{I}-p_{1}}{\mathrm{I}-p_{0}}}+m \frac{\log \frac{\mathrm{I}-p_{0}}{\mathrm{I}-p_{1}}}{\log \frac{p_{1}}{p_{0}}-\log \frac{\mathrm{I}-p_{1}}{\mathrm{I}-p_{0}}}<\mathrm{A}_{\mathrm{m}}<\frac{\log \frac{\mathrm{I}-\beta}{\alpha}}{\log \frac{p_{1}}{p_{0}}-\log \frac{\mathrm{I}-p_{1}}{\mathrm{I}-p_{0}}}+m \frac{\log \frac{\mathrm{I}-p_{0}}{\mathrm{I}-p_{1}}}{\log \frac{p_{1}}{p_{0}}-\log \frac{\mathrm{I}-p_{1}}{\mathrm{I}-p_{0}}}
$$

$$
\begin{aligned}
& \mathrm{A}_{\mathrm{m}} \geq \frac{\log \frac{\mathrm{I}-\beta}{\alpha}}{\log \frac{p_{1}}{p_{0}}-\log \frac{\mathrm{I}-p_{\mathrm{I}}}{\mathrm{I}-p_{0}}}+m \frac{\log \frac{\mathrm{I}-p_{0}}{\mathrm{I}-p_{\mathrm{I}}}}{\log \frac{p_{\mathrm{I}}}{p_{0}}-\log \frac{\mathrm{I}-p_{1}}{\mathrm{I}-p_{0}}}=r_{\mathrm{m}} \\
& \mathrm{A}_{\mathrm{m}} \leq \frac{\log \frac{\beta}{\mathrm{I}-\alpha}}{\log \frac{p_{1}}{p_{0}}-\log \frac{\mathrm{I}-p_{1}}{\mathrm{I}-p_{0}}}+m \frac{\log \frac{\mathrm{I}-p_{0}}{\mathrm{I}-p_{1}}}{\log \frac{p_{1}}{p_{0}}-\log \frac{\mathrm{I}-p_{1}}{\mathrm{I}-p_{0}}}=a_{\mathrm{m}}
\end{aligned}
$$

dove compaiono le due espressioni $a_{\mathrm{m}}$ ed $r_{\mathrm{m}}$ dette rispettivamente «numero di accettazione », e «numero di repulsione »: $a_{\mathrm{m}}$ si rende intero se è necessario prendendo il maggiore intero $<a_{\mathrm{m}}$ ed $r_{\mathrm{m}}$ invece prendendo il minor intero $>r_{\mathrm{m}}$.

Quando avviene perciò che all' mesima osservazione $a_{\mathrm{m}}<\mathrm{A}_{\mathrm{m}}<r_{\mathrm{m}}$ si continua ad osservare altri soggetti; quando $\mathrm{A}_{\mathrm{m}}=r_{\mathrm{m}}$ si sospende l'osservazione e si accetta l'ipotesi $\mathrm{H}_{1}$; quando $\mathrm{A}_{\mathrm{m}}=a_{\mathrm{m}}$ si sospende pure l'osservazione ma accettando l'ipotesi $\mathbf{H}_{\mathbf{o}}$. 
Ora, come si rileva dalle equazioni soprascritte, $a_{\mathrm{m}}$ ed $r_{\mathrm{m}}$ sono funzione lineare del numero di osservazioni $m$. Perciò (fig. I) i punti $\left(m, a_{\mathrm{m}}\right) \mathrm{e}\left(m, r_{\mathrm{m}}\right)$ giacciono su due rette $L_{0}$ e $L_{1}$ le quali, parallele tra loro avendo lo stesso coefficiente angolare e ciascuna con una propria intercetta, delimitano le tre zone di accettazione, di repulsione e di indifferenza.

Tracciare queste rette in base ai valori del coefficiente angolare

$$
s=\frac{\log \frac{\mathrm{I}-p_{0}}{\mathrm{I}-p_{1}}}{\log \frac{p_{1}}{p_{0}}-\log \frac{\mathrm{I}-p_{1}}{\mathrm{I}-p_{0}}}
$$

e delle intercette

$$
\begin{aligned}
& h_{\mathrm{o}}=\frac{\log \frac{\beta}{\mathrm{I}-\alpha}}{\log \frac{p_{1}}{p_{0}}-\log \frac{\mathrm{I}-p_{1}}{\mathrm{I}-p_{0}}} \quad \text { per } L_{\mathrm{o}} \mathrm{e} \\
& h_{1}=\frac{\log \frac{\mathrm{x}-\beta}{\alpha}}{\log \frac{p_{1}}{p_{0}}-\log \frac{1-p_{1}}{\mathrm{I}-p_{0}}} \quad \text { per } L_{1}
\end{aligned}
$$

dove compaiono soltanto le quattro grandezze prefissate $p_{0}, p_{1}, \alpha$ e $\beta$ è tutto quanto si richiede di calcolo per l'esecuzione di questo test.

Segnate queste rette, ad esempio su un foglio di carta millimetrata, si descrive il grafico $\mathrm{A}_{\mathrm{m}}$, funzione di $m$. A partire dal punto dell'ascissa che corrisponde alla prima osservazione si riporta il risultato di questa: se il soggetto è A con un punto o trattino nel senso dell'ordinata, se il soggetto è $O$ nel senso dell'ascissa secondo l'unità di rappresentazione scelta distinguendo con segni diversi i maschi dalle femmine. Così si continua per ogni successiva osservazione. Si termina quando $\left(m, A_{m}\right)$ tocca o passa una delle due rette. Se tocca o passa la retta $L_{0}$ si accetta l'ipotesi $\mathrm{H}_{0}$; se tocca o passa la retta $L_{1}$ si respinge l'ipotesi zero ammettendo la ipotesi alternativa $\mathrm{H}_{1}$ : cioè, nel nostro caso, la prevalenza significativa di soggetti di gruppo A nella popolazione dei malati e quindi l'associazione tra detto gruppo e malattia.

Sarà conveniente eseguire contemporaneamente due test identici, per il gruppo A e per il gruppo $\mathrm{O}$, costruendo il grafico appropriato per ciascuno. Se si desidera si può fare lo stesso anche per gli altri gruppi $\mathrm{B}$ e AB; ma è evidente che la loro bassa frequenza presso di noi impedirà di raggiungere un risultato in tempo ragionevole eccetto che si tratti di malattie molto frequenti. 
I vantaggi di condurre un esperimento in continuazione, senza alcuna fatica eccetto quella di notare il risultato delle osservazioni ogni volta che si presenta l'occasione, di controllare man mano la tendenza del fenomeno, di raggiungere deci-

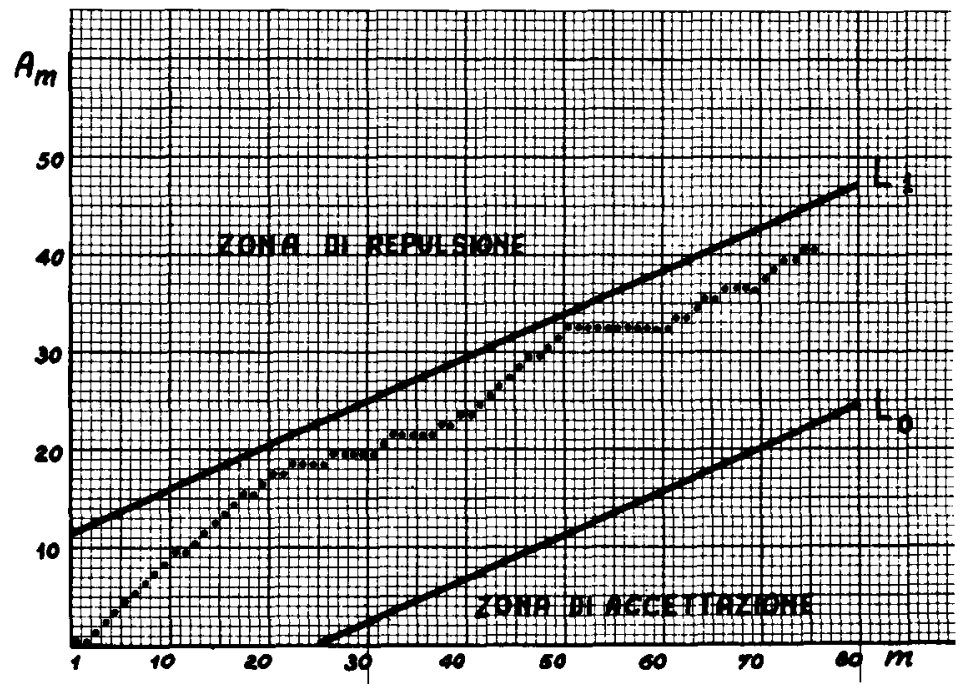

Fig. I. Applicazione del test sequenziale con procedimento grafico ai casi di cancro del collo dell'utero, ricoverati negli anni I 954-1956 presso l'Istituto Nazionale per lo studio e la cura dei Tumori in Milano (Beolchini e coll., I958b), per l'analisi dell'associazione con il gruppo A.

$s=0,45 ; \quad h_{0}=-\mathbf{I} \mathbf{I}, 5 ; \quad h_{\mathbf{1}}=\mathrm{I} \mathbf{I}, 5 \quad p_{0}=0,4 \mathbf{I} 8 ; \quad p_{1}=0,48 \mathrm{I}$

L'osservazione si dovrebbe continuare.

sioni attendibili quanto quelle dei metodi classici con minor numero di osservazioni e di fornire nello stesso tempo i dati richiesti per ulteriori elaborazioni potranno forse orientare all'uso di questo metodo di analisi.

\section{Conclusione}

Allo stato attuale delle ricerche sembra un po' enfatica l'espressione di McConnell (I957): " it is probable that the easiest part of this research is now completed: finding out if there are associations » (p. 20I). E invece desiderabile che questo genere di ricerche nelle popolazioni si estenda non solo in ampiezza, comprendendo un numero sempre maggiore di malattic - soprattutto di quelle che si manifestano con una certa frequenza in giovane età - ed un grande numero di osservazioni per ogni malattia; ma anche in profondità, studiando il fenomeno distintamente nei due sessi, nelle differenti età ed in relazione con altri sistemi di antigeni e con il carattere «secretore» nei liquidi che possono essere particolarmente interessati. 
E però necessario, affinchè le singole ricerche siano conclusive o almeno sufficientemente indicative e possano essere utilizzate in ulteriori analisi, che venga posta una partịcolare attenzione nel primo tempo della ricerca, cioè nel campionamento procurando di evitare selezione insufficienza ed eterogeneità e raccogliendo i dati sui singoli casi con la maggior uniformità possibile di criteri.

\section{Riassunto}

L'importanza genetica e medica della ricerca sulle relazioni tra gruppi sanguigni e malattie nelle popolazioni umane impone una grande accuratezza.

Nel presente lavoro si è sottolineata la necessità che i campioni siano rappresentativi, sufficienti ed omogenei, indicando alcuni elementi capaci di viziare il campionamento sotto l'uno o l'altro di tali aspetti.

Si sono quindi ricordati i metodi classici di analisi, per lo studio di campioni singoli e combinati, secondo Aird, Woolf e Haldane, ponendone in rilievo vantaggi e svantaggi.

Si è infine presentato un metodo sequenziale di facile ed utile applicazione per quanti possono svolgere le indagini direttamente nelle cliniche.

\section{Bibliografia}

Aird I., Bentall H.H. e Roberts J.F.A.: Relation between cancer of the stomach and the ABO blood groups. Brit. Med. J., r, 799-80 r, r953.

Aird I., Mehigan J.A. e Roberts J.A.F.: The blood groups in relation to peptic ulceration and carcinoma of colon, rectum, breast and bronchus. Brit. Med. J., 2, 315-321, 1954 .

Benda N. e Mengrini G.: Correlazioni tra gruppi sanguigni ed incidenza di alcune forme morbose. La Riforma Medica, 7I, I69-172, 1957.

Bentall H. H.: Blood groups and disease with special reference to stomal ulcer and pernicious anemia. Proceed. Ist. Intern. Congress Hum. Genetics, Copenhagen 1956, III, 410-412, I 957.

Beolchini P.E., Cresseri A., De Maria B., Morganti G., Peruzzotti R. e Serra A.: Carcinoma gastrico e gruppi sanguigni del sistema ABO. Analecta Genetica, 6, I I2-I I 7, 1958 a.

Beolchini P. E., Gresseri A., De Maria B., Morganti G., Peruzzotti R. e Serra A.: Rapporti tra neoplasie del collo dell'utero e gruppi sanguigni del sistema ABO (dati preliminari). Analecta Genetica, 6, IO9-111, I $958 \mathrm{~b}$.

Beolchini P.E.: Comunicazione personale.

Billington B.P.: Gastric cancer relationships between ABO blood groups site and epidemiology. Lancet (London) I, 859-862, 1956 .

Brown D.A.P., Merrose A.G. e Wallace J.: The blood groups in peptic ulceration. Brit. Med. J., 2, I35-137, I956.

Buckwalter J.A. e Knowler L.A.: Blood donor controls for blood group disease researches. Am. J. Hum. Genetics, 10, I64-I 74, $195^{8}$.

Buckwalter J.A., Wohlwend E.B., Colter D.C. e Tidrick R.T.: Natural selection associated with the ABO blood group. Science, 123, 840, 1956a.

Buckwalter J.A., Wohlwend E.B., Colter D.C., Tidrick R.T. e Knowler L.A.: ABO blood groups and disease. J.A.M.A., 162, $1210-1215,1956 \mathrm{~b}$.

Cappellini E. e Gupfanti A.: Gruppi sanguigni e neoplasie. Inchiesta su 449 casi di tumori gastrici e 248 casi di tumori broncopolmonari. Rass. ital. Chir. Med., 5, 555-565, 1956. 
Cavallini R.: Gruppi sanguigni e cancro dell'utero. Il sangue, 3i, 100-104, 1958.

Comitato per lo studio della distribuzione geografica dei gruppi sanguigni in Italia: Schema della distribuzione geografica dei gruppi sanguigni in Italia. Atti del VII Congresso della Società internazionale della Trasfusione del Sangue, Roma $195^{8}$ (in corso di pubblicazione).

Cresseri A. e Serra A.: Sull'applicazione di tests sequenziali nell'analisi dei rapporti tra gruppi sanguigni e neoplasie. Intervento al II Symposium internazionale di Genetica Medica. Torino I957.

Di Carlo G. e Ridulfo S.: Gruppi sanguigni, carcinoma dello stomaco ed ulcera gastro-duodenale. La sintesi, I, 1957 (estratto).

Hat.dane G.B.S.: The estimation and significance of the logarithm of a ratio of frequencies. An. Hum. Gen., 20, 309-3I I, 1956.

HogG L. Jr., PAck G.T.: The controversial relationship between blood group A and gastric cancer. Gastroenterology, 32, 797-805, 1957.

Jennings D., Balme R.H. e Richardson J.E.: Carcinoma of stomach in relation to ABO blood groups. Lancet (London), 2, I I-I 2, I956.

Liotta I., Frattaroli W., e Herzel A.: Rapporti tra carcinoma dell'utero e gruppi sanguigni. Riv. Emoter. Immunematol., 4, 205-209, 1957.

Maltoni G. e Canali E.: I gruppi sanguigni in relazione al cancro gastrico e all'ulcera gastroduodenale. Ann. it. Chirurgia, $33,37 \mathrm{I}-3^{82}$, 1956 .

Mangani G.: Della relazione esistente tra gruppi sanguigni ed alcune affezioni chirurgiche. Il Policlinico, 64 , I049-1052, 1957 .

Manuila A.: Recherches sérologiques et anthropologiques chez les populations de la Roumanie et des régions voisines. Contribution à l'étude du problème dinarique. Archiv. J. Klaus-Stiftung, 32, $219-357,1957$.

MAnulla A.: Blood groups and disease. Hard facts and delusions. J.A.M.A. 167, 2047-2052, I958.

McConnell R.B.: Blood groups and disease. A promising field of medical research. Eug. Quart., 4, I97-201, 1957.

Morganti G., Avanza G., Barbaini S. e Granotti G. A.: Distribuzione dei gruppi e dei tipi sanguigni in Italia. La trasfusione del sangue, 2, 43-57, 1957 .

Pollara C. e Melina D.: Gruppi sanguigni e malattie. La Riforma Medica, 71, 929-930, 1957.

Roberts J. A. F.: Associations between blood groups and discase. Copia ciclostilata della Comunicazione al IIe Congrès national de Transfusion sanguine - Bordeaux 1956 .

Roberts J.A.F.: Associations between blood groups and disease. Proc. 1st. Intern. Congress Hum. Genetics. Copenhagen 1956, III, 393-404, 1957.

Roberts J.A.F.: Gruppi sanguigni e suscettibilità alle malattie. Recenti progressi in medicina, 25, I-39, I958.

Spandonari A. e Repetto Carboneschi W.: Correlazioni statistiche dei gruppi sanguigni con l'ulcera gastroduodenale e con il carcinoma dello stomaco. Rass. it. Chir. Med., 6, I I3-117, I957.

SpeISER P.: Bestehen mathematisch gesicherte Beziehungen der ABO-Gruppen, des Rhesusfaktors $R h_{o}$ (D) und des Geschlechtes zu Carcinoma ventriculi, Ulcus ventriculi und Ulcus duodeni? Der Krebsarzt, II, $344-348,1956$.

Wald A.: Sequential Analysis. Wiley, New York, i947.

Wallace J.: Blood groups and disease. Brit. Med. J., 2, 534, I954.

Walther W. W., Raeburn C. e Case J.: Blood groups in relation to malignant diseases. Lancet (London), I, $970-972,1956$.

Woolf B.: On estimating the relation between blood groups and disease. Ann. Hum.Genetics, 19, 25 I-253, I955. 


\section{RÉSUMÉ}

L'importance génétique et clinique des recherches sur les relations entre groupes sanguins et maladies dans les populations humaines exige un grand soin.

Pour cette raison on a souligné ici la nécessité que les échantillons soient représentatifs. suffisants et homogènes et on a indiqué quelques éléments qui peuvent vicier l'échantillonnage sous l'un ou l'autre de tels aspects.

On a depuis mentionné les méthodes classiques d'analyse, sur les échantillons simples et combinés, selon Aird, Woolf et Haldane, en relevant les avantages et les désavantages.

On a enfin présenté une méthode séquenciale qui peut être très facilement et utilement appliquée par ceux qui font la recherche directement dans les cliniques.
SUMMARY

The genetical and medical importance of the research on the relations between blood groups and disease in the human populations requires a notable caution.

In the present paper the need is pointed out that the samples be representative, sufficient and homogeneous, and some elements are noted which might introduce sampling biases.

Then the classic methods of analysis according to Aird, Woolf and Haldane are mentioned and their advantages and disadvantages outlined.

Finally a sequential method of analysis is presented that may be applied very easily and usefully by the researchers who might have the data directly and continuously in the hospitals.

\section{ZUSAMMENFASSUNG}

Dic Bedeutung, in genetischer und klinischer Hinsicht, der Untersuchungen über die Zusammenhänge zwischen Blutgruppen und Krankheiten unter verschiedenen Bevölkerungen fordert grösste Sorgfalt.

In der vorliegenden Arbeit wird besonders die Notwendigkeit betont, dass die Muster wirklich repräsentativ, genügend und homogen seien, und es wurde auch auf einige Faktoren hingewiesen, die die Muster unter der einen oder anderen Hinsicht verderben könnten.

Es werden folglich die klassischen Analysemethoden nach Aird, Woolf und Haldane angeführt, bestimmt für Untersuchungen von Einzelmuster und Musterkombinazionen, und es wurden Vorteile und Nachteile hervorgehoben.

Es wird endlich eine Sequenzialmethode entwickelt, die leicht und mit Nutzen von denen angewendet werden kann, die die Untersuchungen direkt und andauernd in den Kliniken anstellon können. 